\title{
DOUBLE-DIFFUSIVE NATURAL CONVECTION OF LOW PRANDTL NUMBER LIQUIDS WITH SORET AND DUFOUR EFFECTS
}

\author{
Gang Qiu ${ }^{\mathrm{a}}$, Mo Yang,", Jin Wang ${ }^{\mathrm{b}}$, Yuwen Zhang \\ ${ }^{a}$ School of Energy and Power Engineering, University of Shanghai for Science and Technology, Shanghai, 200093, China \\ ${ }^{b}$ College of Aeronautics and Astronautics, Shanghai Jiao Tong University, Shanghai 200240, China \\ ${ }^{c}$ Department of Mechanical \& Aerospace Engineering, University of Missouri, Columbia, Missouri, MO 65211, USA
}

\begin{abstract}
An unsteady numerical model for double-diffusive natural convection of low Prandtl number liquids with Soret and Dufour effects inside the horizontal cavity is developed. The thermosolutal model is solved numerically using the SIMPLE algorithm with QUICK scheme. The flow field, temperature and concentration distributions for different buoyancy ratios, Rayleigh numbers and aspect ratios under different Prandtl numbers are studied systematically. The results reveal that the flow structure develops from conduction-dominated to convection as buoyancy ratio increases under different Prandtl numbers. Heat transfer intensity keeps constant and mass transfer intensity grows slowly before a critical point as Rayleigh number increases for different Prandtl numbers. Then heat transfer intensity enhances dramatically and mass transfer intensity increases faster as Rayleigh number increases after a critical point. The vortex number of flow structure, recirculation zones of isotherm and isoconcentration contours, heat and mass transfer intensity increase as aspect ratio decreases under different Prandtl numbers.
\end{abstract}

Keywords: Double-diffusive natural convection; low Prandtl numberliquids; Soret effect; Dufour effect; different Prandtl numbers

\section{INTRODUCTION}

Liquid metals (low Prandtl number liquids) have drawn attentions from researchers due to their wide applications in various fields such as nuclear reactors (Kang et al., 2016; Kang et al., 2013; Efanov et al., 2007; El-Genk and Tournier, 2011), crystal growth (Akour and Jarrah, 2005), semiconductor manufacturing process (Eslamia and Saghir, 2011), heat dissipation of electronic devices (Ma and Liu, 2007) and other fields. The flow condition of natural convection of liquid metal have impacts on the heat transfer or mass transfer of the whole process.

There are various studies about natural convection of the pure liquid metal with numerical and experimental methods. Cao and Faghri (1990) numerically studied natural convection of fluids having very low Prandtl numbers down to $\mathrm{Pr}=0.00125$ in rectangular enclosures with partial heating from below. Cless and Prescott (1996) studied the effects of timevarying thermal boundary conditions on oscillatory natural convection of liquid metals in a differentially heated square cavity. Moh et al. (1997) analyzed the interaction between buoyancy-induced convection within a liquid metal inside a square, differentially heated enclosure and externally imposed excitation in the form of harmonic rocking about the enclosure center point. Zhang and Angeli (2011) investigated the combined thermally and electromagnetically driven natural convection of a liquid metal heated by Joule effect in a rectangular cavity to figure out the flow structure transitions. Sharma et al. (2009) investigated turbulent natural convection of liquid metal in a cylindrical enclosure with locally distributed heat source. Karcher et al. (2002) used numerical and experimental methods to study the heat transfer of natural convection with liquid metal inside the magnetic field. Mahfooz et al. (2012) studied the radiation effects on a transient two-dimensional natural convection of a laminar boundary-layer flow with viscous incompressible electrically conducting and optically dense fluid along a vertical flat surface with heat generation. Campo et al. (2006) numerically investigated laminar natural convection of metallic fluids between vertical parallel plate channels with isoflux heating. Man et al. (2000) conducted comprehensive numerical simulation to investigate three-dimensional, steady, conjugate heat transfer of natural convection and conduction in a vertical cubic enclosure which contained a centered, cubic, conducting heat source. Bucchignani and Mansutti (2000) carried out a numerical analysis of the buoyancy-driven and unsteady natural convection of a liquid metal in a three dimensional shallow cavity $(4 \times 1 \times 1)$ with a horizontal temperature gradient along the larger dimension and observed the Ruelle-Takens bifurcation. Li et al. (1997) conducted a threedimensional unsteady computation to investigate buoyancy-driven unstable convection in a side-wall-heated and top-wall-cooled vertical closed cylinder containing liquid metal.

In fact, thermal and solutal buoyancies can induce many intriguing and complex flow and heat transfer phenomena during the doublediffusive natural convection of liquid metals or liquid mixtures. Therefore, many scholars showed interest in the process. Hyun et al. (1995) simulated two-dimensional double-diffusive natural convection in $\mathrm{Al}-\mathrm{Cu}$ and $\mathrm{Pb}-\mathrm{Sn}$ liquid metals systems using a Chebyshev collocation (spectral) method. Siddiqa et al. (2017) analyzed the influence of heat and mass transfer on double-diffusive natural convection boundary layer flow of thermally radiating wavy surface. Prescott and Incropera (1994) simulated the solidification of a Pb-19\% Sn alloy in an annular test cell subjected to axisymmetric cooled along its outer vertical wall and the simulation demonstrated the importance of thermosolutal convection during solidification. Yin and Koster (1999) studied stratification of indium and the convective flows during solidification of Ga-5\% In alloy fluid, and analyzed the influence of natural convection on the solid-liquid interface morphology. Akour and Jarrah (2005) conducted numerical and experimental investigations on the solidification process of aluminum$5.5 \%$ copper binary alloy in an axisymmetric metal-mold cooled in the

* Corresponding author. Email: yangm@usst.edu.cn 
lab atmosphere, and studied the effect of natural convection on the solidification process and the alloy structure.

Mass transfer due to a temperature gradient in a double-diffusive convection of liquid mixtures is called thermodiffusion, or Soret effect. And heat transfer due to a concentration gradient in a double-diffusive convection of liquid mixtures is called Dufour effect. There is Soret effect during manufacturing process of some semiconductor materials with the temperature zone melting method (TZGM). Eslamian and Saghir (2011) studied the Soret effect in a binary molten semiconductormetal layer with TZGM, estimated themodiffusion factor, and simulated convection of molten zone in a two-dimensional rectangular cell heated at the top or bottom with various thicknesses and aspect ratios. JafarSalehi (2014) et al. proposed an expression for the estimation of themodiffusion factor in the liquid metal alloys fluid, and investigated thermosolutal natural convection numerically in a two-dimensional cavity filled with $\mathrm{Sn}-\mathrm{Bi}$ alloy whose sidewalls exposed to surface radiation and external convection.

However, there are fewer studies about double-diffusive natural convection of liquid metals with Soret and Duffor effect inside a horizontal cavity. Therefore, an unsteady numerical model based on thermosolutal buoyancies with Soret and Dufour effects for doublediffusive natural convection of $\mathrm{Pb}$-Sn alloy liquids within the horizontal cavity is developed. The flow field, temperature and concentration distributions for different buoyancy ratios, Rayleigh numbers, and aspect ratios under different Prandtl numbers are investigated systematically.

\section{Physical Model and Governing Equations}

Figure 1 shows the physical model for double-diffusive convection in horizontal cavity due to thermal and solutal buoyancies. The cavity of height $\mathrm{H}$ and width $\mathrm{W}$ (aspect ratio $\mathrm{A}=\mathrm{H} / \mathrm{W}$ ) is filled with binary metal fluid of $\mathrm{Pb}$ and $\mathrm{Sn}$ which have a uniform temperature $T_{0}$ and concentration $c_{0}$. The bottom wall is fully heated and is maintained at the high temperature and concentration of $T_{h}$ and $c h$, respectively. The upper wall is subject to the uniform temperature and concentration of $T_{l}$ and $c l$, respectively. The double-diffusive convection occurs under the vertical temperature and concentration gradients. The two side-walls of the cavity are adiabatic and impermeable. The walls apply to non-slippery condition.

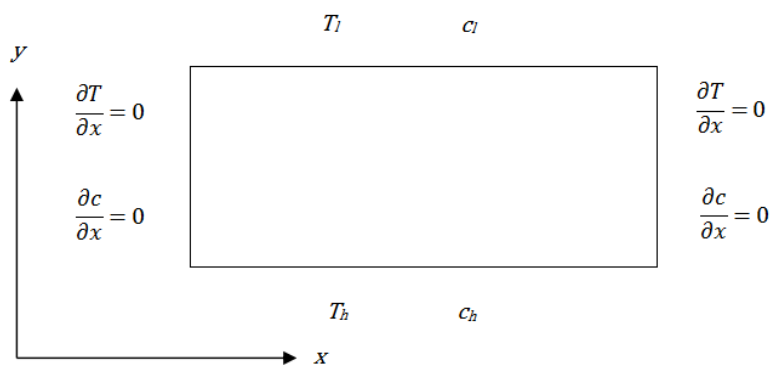

Fig. 1 Physical model for double-diffusive convection

The double-diffusive natural convection is in two-dimensional unsteady state and it is assumed that there is no heat generation, chemical reactions, and thermal radiation. The binary fluid inside the cavity is Newtonian fluid. All thermophysical properties of the medium are constants except for density in the buoyancy term based on the Boussinesq approximation:

$\rho=\rho_{0}\left[1-\beta_{T}\left(T-T_{0}\right)-\beta_{c}\left(c-c_{0}\right)\right]$

where $\rho_{0}$ is the medium density at the reference temperature $T_{0}$ and concentration $c_{0} . \beta_{T}$ and $\beta_{c}$ are the thermal and solute volumetric expansion coefficients, respectively.

The conservation equations for laminar double-diffusive convection in horizontal cavity with Soret and Dufour effects can be expressed just like those in the paper of Wang et al. (2015). Then conservation equations are transformed into the dimensionless format:

$$
\begin{aligned}
& \frac{\partial U}{\partial X}+\frac{\partial V}{\partial Y}=0 \\
& \frac{\partial U}{\partial \tau}+U \frac{\partial U}{\partial X}+V \frac{\partial U}{\partial Y}=-\frac{\partial P}{\partial X}+\operatorname{Pr}\left(\frac{\partial^{2} U}{\partial X^{2}}+\frac{\partial^{2} U}{\partial Y^{2}}\right) \\
& \frac{\partial V}{\partial \tau}+U \frac{\partial V}{\partial X}+V \frac{\partial V}{\partial Y}=-\frac{\partial P}{\partial Y}+\operatorname{Pr}\left(\frac{\partial^{2} V}{\partial X^{2}}+\frac{\partial^{2} V}{\partial Y^{2}}\right)+ \\
& R a \cdot \operatorname{Pr}(\theta+N c C) \\
& \frac{\partial \theta}{\partial \tau}+U \frac{\partial \theta}{\partial X}+V \frac{\partial \theta}{\partial Y}=\left(\frac{\partial^{2} \theta}{\partial X^{2}}+\frac{\partial^{2} \theta}{\partial Y^{2}}\right)+D_{f}\left(\frac{\partial^{2} C}{\partial X^{2}}+\frac{\partial^{2} C}{\partial Y^{2}}\right) \\
& \frac{\partial C}{\partial \tau}+U \frac{\partial C}{\partial X}+V \frac{\partial C}{\partial Y}=\frac{1}{L e}\left[\left(\frac{\partial^{2} C}{\partial X^{2}}+\frac{\partial^{2} C}{\partial Y^{2}}\right)+S_{r}\left(\frac{\partial^{2} \theta}{\partial X^{2}}+\frac{\partial^{2} \theta}{\partial Y^{2}}\right)\right]
\end{aligned}
$$

where the definitions of above dimensionless parameters can be seen as follows:

$$
\begin{aligned}
& (U, V)=\frac{(u, v) W}{\alpha},(X, Y)=\frac{(x, y)}{W}, \theta=\frac{T-T_{l}}{T_{h}-T_{l}}, C=\frac{c-c_{l}}{c_{h}-c_{l}}, \\
& \tau=\frac{\alpha t}{W^{2}}, P=\frac{W^{2}\left(p+\rho_{0} g y\right)}{\rho_{0} \alpha^{2}}, P r=\frac{v}{\alpha}, L e=\frac{\alpha}{D^{\prime}} \\
& R a=\frac{g W^{3} \beta_{T}\left(T_{h}-T_{l}\right)}{v \alpha}, N c=\frac{\beta_{c}\left(c_{h}-c_{l}\right)}{\beta_{T}\left(T_{h}-T_{l}\right)}, D_{f}=\frac{\kappa_{T C}\left(c_{h}-c_{l}\right)}{\alpha\left(T_{h}-T_{l}\right)}, \\
& S_{r}=\frac{\kappa_{C T}\left(T_{h}-T_{l}\right)}{D\left(c_{h}-c_{l}\right)}
\end{aligned}
$$

The dimensionless controlling parameters are the buoyancy ratio Nc, Prandtl number Pr, Rayleigh number Ra, Lewis number Le, Dufour coefficient Df and Soret coefficient $\mathrm{Sr}$.

The boundary and initial conditions turn to be:

$$
\begin{aligned}
& X=0, U=V=0, \frac{\partial \theta}{\partial X}=0, \frac{\partial C}{\partial X}=0 \\
& X=1, U=V=0, \frac{\partial \theta}{\partial X}=0, \frac{\partial C}{\partial X}=0 \\
& Y=0, U=V=0, \theta=1, C=0 \\
& Y=A, U=V=0, \theta=1, C=0 \\
& \tau=0, U=V=0, \theta=0, C=0
\end{aligned}
$$

To characterize heat and mass transfer near the upper and lower side in horizontal cavity, the local Nusselt number $\mathrm{Nu}$ and Sherwood number Sh are defined by:

$$
\begin{aligned}
N u(X) & =\left.\frac{\partial \theta}{\partial Y}\right|_{Y=0}+\left.D_{f} \frac{\partial C}{\partial Y}\right|_{Y=0} \\
S h(X) & =\left.\frac{\partial C}{\partial Y}\right|_{Y=0}+\left.S_{r} \frac{\partial \theta}{\partial Y}\right|_{Y=0}
\end{aligned}
$$

where the first and second term of Eq. (12) indicate the Fourier's heat flux and the diffusion thermo flux due to Dufour effect, respectively. The first and second term in Eq. (13) characterize the Fick's diffusion flux and the thermodiffusion flux induced by Soret effect, respectively.

And the average Nusselt and Sherwood numbers can be expressed as:

$$
\begin{gathered}
\overline{N u}=\int_{0}^{1} N u(X) d X \\
\overline{S h}=\int_{0}^{1} \operatorname{Sh}(X) d X
\end{gathered}
$$

\section{NUMERICAL SOLUTION}

The dimensionless Navier-Stokes equations are numerically solved on a staggered grid system, then the discretized equations are solved by SIMPLE algorithm based in the finite volume method. Euler backward 
second-order implicit scheme is employed for the unsteady term. The convective term was discretized using the QUICK scheme. During the SIMPLE iteration process, the pressure correction method taking the effect of concentration into consideration is established in order to obtain the real velocity field. The under-relaxation method is adopted to ensure the convergence of the numerical results and the criterion on one time step is established as the following:

$$
\sum_{i, j}\left|\phi_{i, j}^{n}-\phi_{i, j}^{n-1}\right|<10^{-6}
$$

where $\Phi$ is the generic variable which can be $U, V, \theta$, or $C$, and the superscript $\mathrm{n}$ indicates the iteration number in each time step. The subscript sequence $(i, j)$ represents the grid node.

The grid independence research was conducted under different conditions. Three different non-uniform girds, namely, $150 \times 50,150 \times 80$ and $200 \times 100$ are employed to simulate the double-diffusive convection in the horizontal cavity. The non-uniform grid of $150 \times 80$ is good enough to ensure the grid independent solution when computation accuracy and CPU time are taken into account in the range of variables. So this $150 \times 80$ grid is employed for all subsequent simulations. Meanwhile, the time step independence has been examined and the dimensionless time step of 0.01 is applied.

\section{RESULTS AND DISCUSSIONS}

In order to verify the numerical code for the heat and mass transfer phenomena of the low Prandtl number with the Soret and Duffor effects, natural convection of pure metal liquid in the cavity is simulated first, which is studied by $\mathrm{Li}$ et al. (2016). The dimensionless control parameters $\mathrm{Nc}$, Df and $\mathrm{Sr}$ are all set to 0 , then Le is 1 . The concentration inside the cavity is 0 . Table 1 shows the results is in good agreement with Li's results.

Table 1 Comparison of results between Li's study and present paper in low Pr pure liquid metal

\begin{tabular}{|l|l|l|l|l|}
\hline $\mathrm{Pr}$ & $\mathrm{Ra}$ & $\mathrm{Li}$ & Present study & Deviation $(\%)$ \\
\hline 0.01 & $10^{4}$ & 1.95 & 1.948 & $0.39 \%$ \\
\hline 0.01 & $5 \times 10^{4}$ & 2.8 & 2.786 & $0.5 \%$ \\
\hline 0.005 & $10^{5}$ & 2.65 & 2.673 & $-0.87 \%$ \\
\hline
\end{tabular}

The second verification case is double-diffusive the heat and mass transfer without Soret and Duffor effects under different Nc, which has been studied by Béghein (1992). The dimensionless parameters Df and $\mathrm{Sr}$ are set to 0 . Then Le is 1 , and $\mathrm{Pr}$ is 0.71 . Nc ranges from -0.01 to -5 . Table 2 shows that the relative deviation between the results of present study and Béghein's is within computational uncertainty.

Table 2 Comparison of results between Béghein's study and present paper for the double-diffusive the heat and mass transfer phenomena

\begin{tabular}{|l|l|l|l|}
\hline Nc & Beghein & Present study & Deviation $(\%)$ \\
\hline-0.01 & 16.4 & 16.511 & $0.68 \%$ \\
\hline-0.1 & 16 & 16.106 & $0.66 \%$ \\
\hline-0.2 & 15.5 & 15.616 & $0.75 \%$ \\
\hline-0.5 & 13.6 & 13.788 & $1.38 \%$ \\
\hline-0.8 & 10.6 & 10.757 & $1.48 \%$ \\
\hline-0.9 & 8.8 & 8.8748 & $0.85 \%$ \\
\hline-1.5 & 13.6 & 13.788 & $1.38 \%$ \\
\hline-5 & 23.7 & 23.483 & $-0.92 \%$ \\
\hline
\end{tabular}

Figure 2 shows the steady-state contours of temperature and concentration for $\mathrm{A}=0.5, \mathrm{Nc}=-2.0, \mathrm{Ra}=2000$, and $\mathrm{Sr}=\mathrm{Df}=0.1, \mathrm{Pr}=$ 0.02 . The contours show that the isotherms and isoconcentration contours are parallel to the horizontal walls and the spacing between the contours is constant. This indicates that the heat and mass transfer uniformly and vertically upward. Further study reveals that the velocity of the fluid inside the entire cavity is almost zero. The $\mathrm{Nc}$ in this case equals to -2.0 , meaning that the upward solutal buoyancy is larger than the downward thermal buoyancy which leads the double-diffusive convection approaches to a conduction-dominated regime. More numerical simulations show that when $\mathrm{Nc}$ is less than 0 , the heat and mass transfer remains in conduction-dominated state.

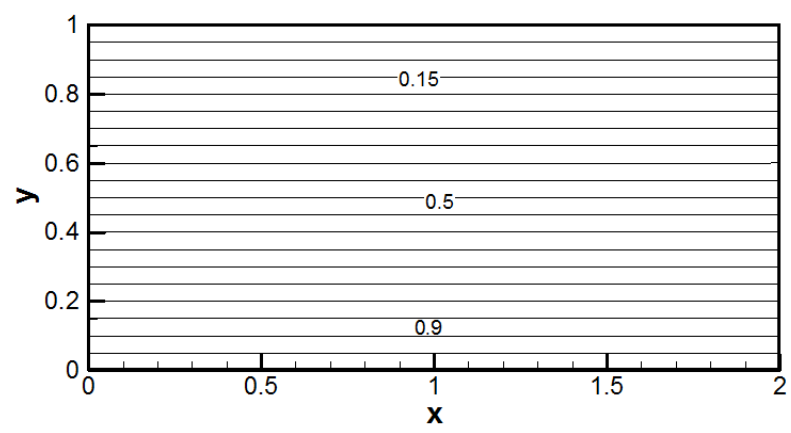

(a) contours of temperature

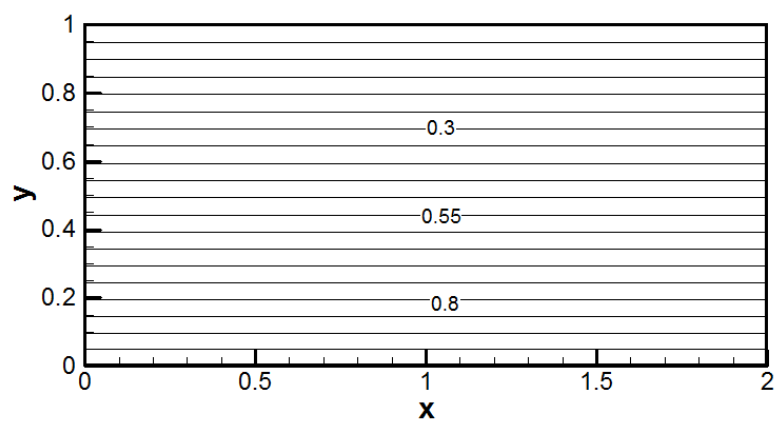

(b) contours of concentration

Fig. 2 Conduction-dominated solution for $\mathrm{A}=0.5, \mathrm{Nc}=-2.0, \mathrm{Ra}=2000$, and $\mathrm{Sr}=\mathrm{Df}=0, \mathrm{Pr}=0.02$

When $\mathrm{Nc}$ increases to 0.03 , it transforms to the oscillatory convection-dominated state. Fig. 3 shows oscillatory convectiondominated solutions of concentration distributions for $\mathrm{A}=0.5, \mathrm{Nc}=0.03$, $\mathrm{Ra}=2000$, and $\mathrm{Sr}=\mathrm{Df}=0.1, \mathrm{Pr}=0.02$. It can be observed that contours change periodically as time progresses. As shown in the Fig. 3, the low concentration boundary layer near the middle of the upper wall extends downwardly and then retracts. Then the concentration contours in the middle remain parallel to the horizontal walls for some time. After that, the low concentration boundary layer extends downwardly again. It can also be found that the entire flow field experiences oscillatory changes. Further study shows that while $\mathrm{Ra}$ is relatively low and $\mathrm{Nc}$ is very small which is close to zero (i.e., the thermal and solutal buoyancies are very weak), the fluid remains in the oscillatory convection-dominated state due to Soret and Duffor effects.

Then Nc continually increased to $0.25(\mathrm{~A}=0.5, \mathrm{Ra}=2000, \mathrm{Sr}=\mathrm{Df}$ $=0.1, \operatorname{Pr}=0.02)$, the fluid transforms into in steady-state convectiondominated state. The temperature, concentration and velocity distributions are shown in Fig. 4.

As shown in the Fig. 4, the fluid in the center point near the lower wall flow upward due to the buoyancies. Then it reaches the upper wall, flows along the walls and eventually returns to lower wall, forming two vortexes. From the figure it can also be seen that the lower concentration boundary layer near the middle extends upward and splits into two parts 
when reaching the upper wall. Then the sickle-like concentration blocks forms, whose concentration differs from than that in core of the vortexes. And the concentration contours of two sides are not centered on the $\mathrm{x}=1$. Fig. 5 shows steady-state convection-dominated solutions of temperature, concentration and velocity distributions for $\mathrm{A}=0.5, \mathrm{Nc}=0.5, \mathrm{Ra}=2000$, and $\mathrm{Sr}=\mathrm{Df}=0.1, \operatorname{Pr}=0.02$. It can be observed that the fluids in the two lower corners flow upward due to the buoyancies. Then it reaches the upper wall, flows along the walls and eventually returns to lower wall, forming two vortexes whose direction are different from that in the case for $\mathrm{Nc}=0.25$ (Fig. 4).

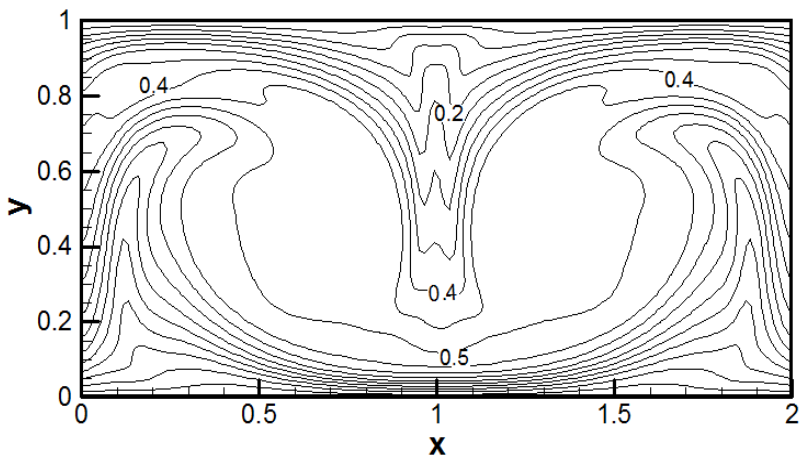

(a) $\tau=4982.41$

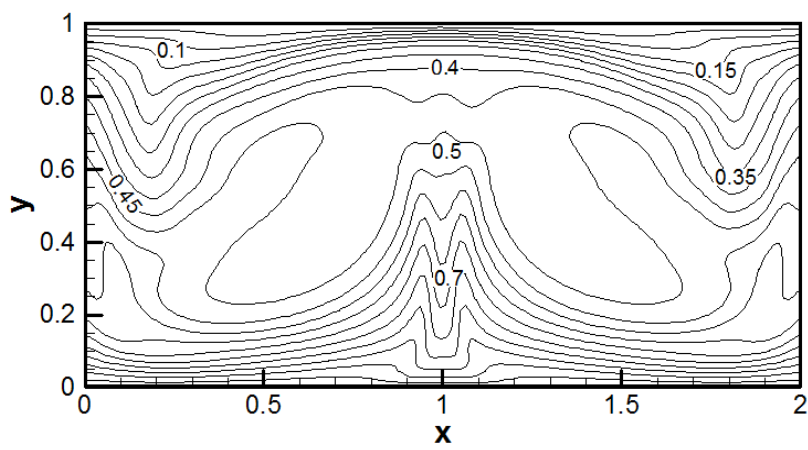

(b) $\tau=4990.222$

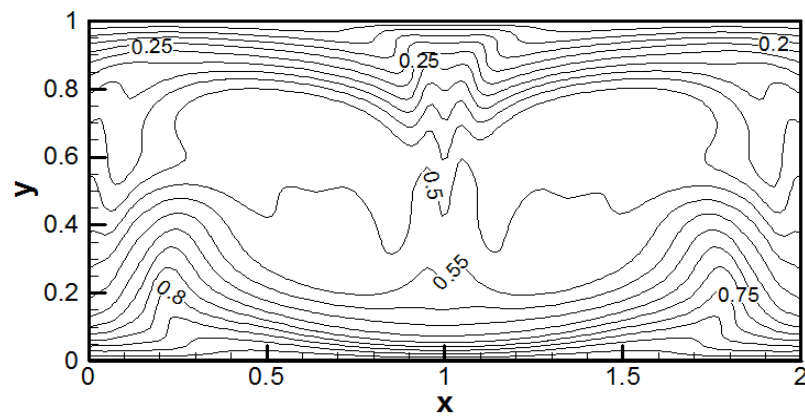

(c) $\tau=4998.034$

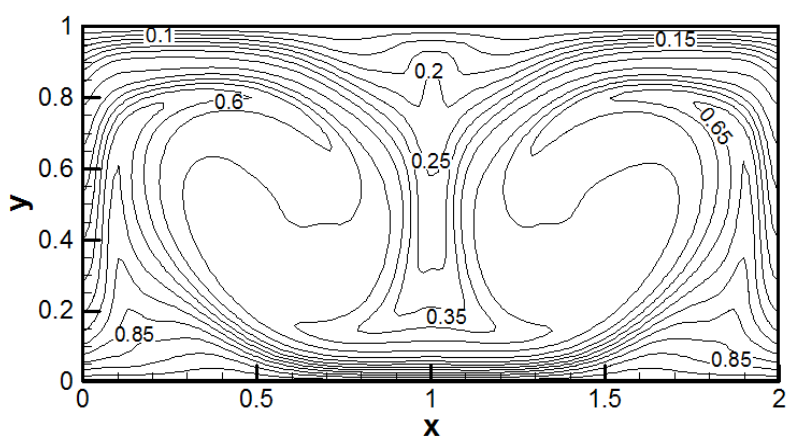

(d) $\tau=5001.94$

Fig. 3 Oscillatory solution for $\mathrm{A}=0.5, \mathrm{Nc}=0.03, \mathrm{Ra}=2000$, and $\mathrm{Sr}=$ $\mathrm{Df}=0.1, \operatorname{Pr}=0.02$

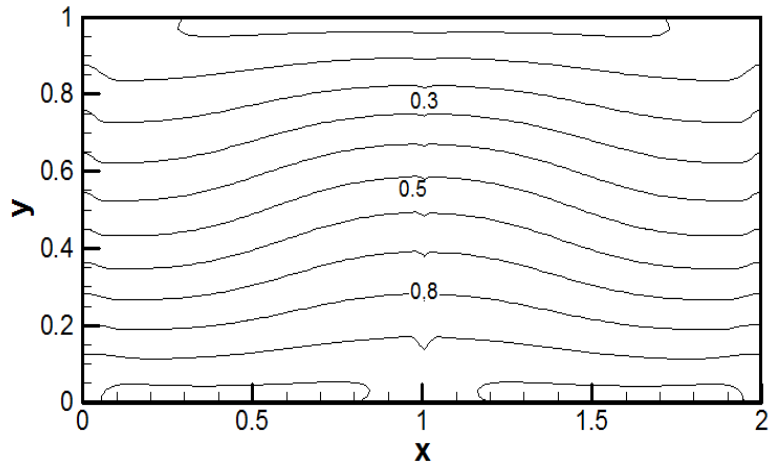

(a) contours of temperature

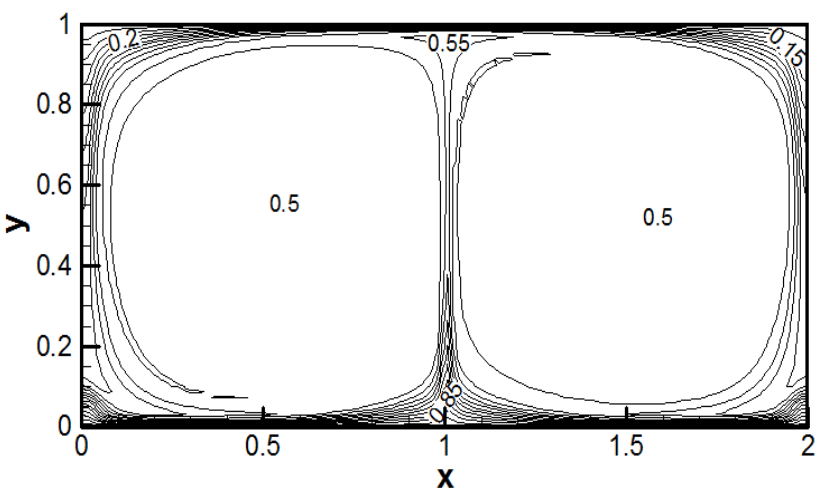

(b) contours of concentration

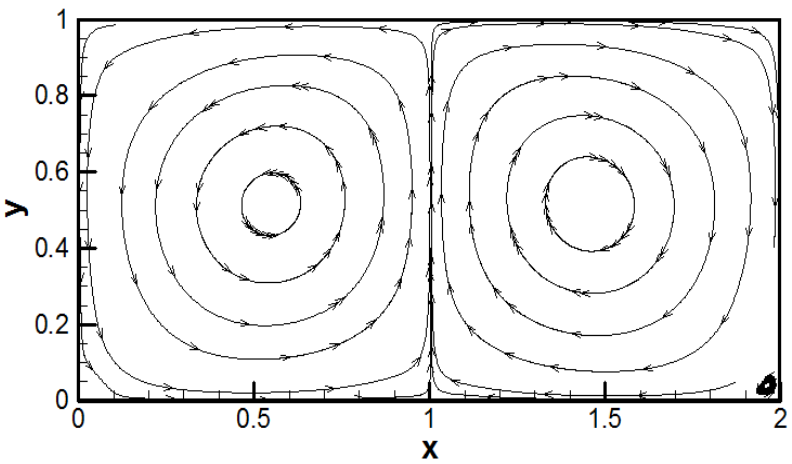

(c) flow field

Fig. 4 Convection-dominated solution for $\mathrm{A}=0.5, \mathrm{Nc}=0.25, \mathrm{Ra}=2000$, $\mathrm{Sr}=\mathrm{Df}=0.1, \mathrm{Pr}=0.02$

When Nc changes from 0.25 to 1.5 , the fluid transforms into the oscillatory convection-dominated state, which is shown in Fig. 6. The concentration distribution changes periodically with time. The upper concentration boundary layer near the middle extends downward and splits into two parts when reaching the lower wall. Then the sickle-like concentration blocks (concentration boundary layer) develops along the vertical walls. In the end, the blocks mix with major concentration block and disappear. While $\mathrm{Nc}$ increases to high value, the oscillatory behaviors show inside the cavity, and the temperature, concentration and vector distributions are asymmetrical.

When Pr changes to 0.015 , or lower values like 0.01 and 0.005 from the previous 0.02 for $\mathrm{Ra}=2000, \mathrm{Nc}=0.5 \mathrm{~A}=0.5$ and $\mathrm{Sr}=\mathrm{Df}=0.1$, the flow structure and concentration distributions are very similar. The result shows that the heat and mass transfer intensity decrease when $\mathrm{Pr}$ decreases. 


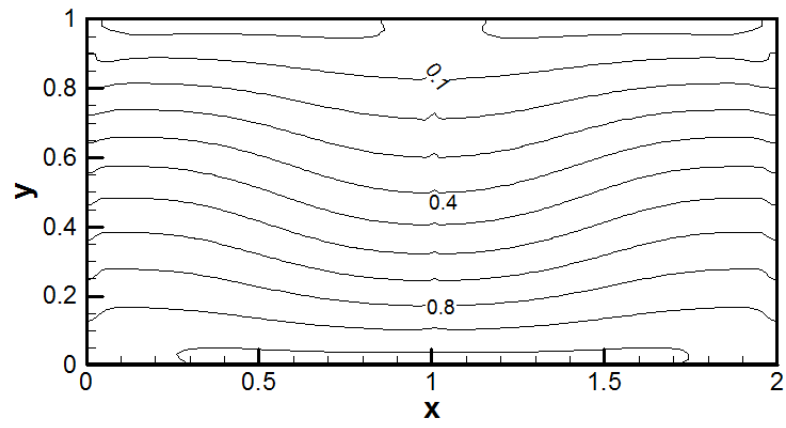

(a) contours of temperature

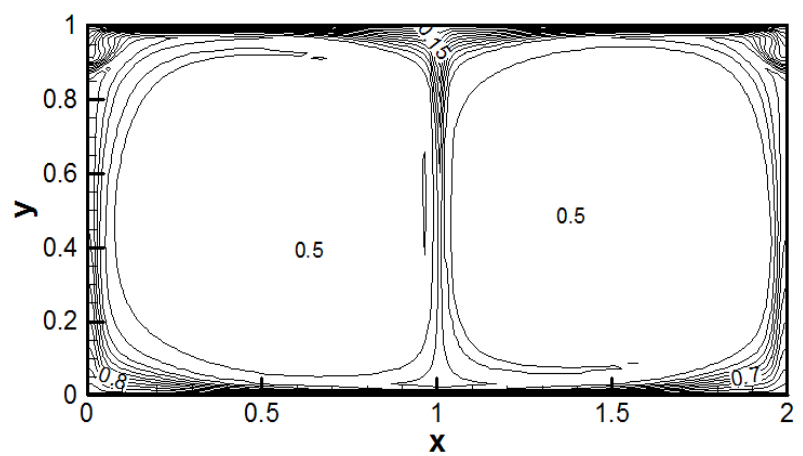

(b) contours of concentration

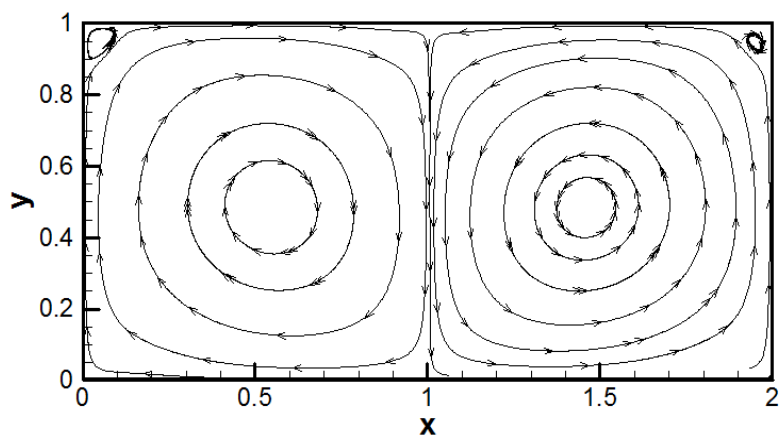

(c) flow field

Fig. 5 Convection-dominated solution for $\mathrm{A}=0.5, \mathrm{Nc}=0.5, \mathrm{Ra}=2000$, $\mathrm{Sr}=\mathrm{Df}=0.1, \mathrm{Pr}=0.02$

Fig. 7 presents effects of buoyancy ratio on double-diffusive natural convection in horizontal cavity under different Pr numbers for $\mathrm{Ra}=2000$, $\mathrm{A}=0.5$ and $\mathrm{Sr}=\mathrm{Df}=0.1$. It demonstrates that both average $\mathrm{Nu}$ and $\mathrm{Sh}$ keep constant firstly and then increase with Nc. There is a transition stage where average $\mathrm{Nu}$ and $\mathrm{Sh}$ bump up because downward resultant force of the thermal and solutal buoyancies becomes larger as increasing Nc and makes the flow structure transform from conduction-dominated structure into convection dominated structure. Besides, it can be observed that $\mathrm{Nu}$ and $\mathrm{Sh}$ rise simultaneously with $\mathrm{Sr}$ and $\mathrm{Df}$ as the coupling-diffusive interaction builds. It can also be seen that $\mathrm{Nu}$ and $\mathrm{Sh}$ becomes higher as Pr increases from 0.005 to 0.02 for the same buoyancy ratio Nc. But the differences of mass transfer intensity becomes smaller between all kinds of low Pr fluid when Nc becomes higher.

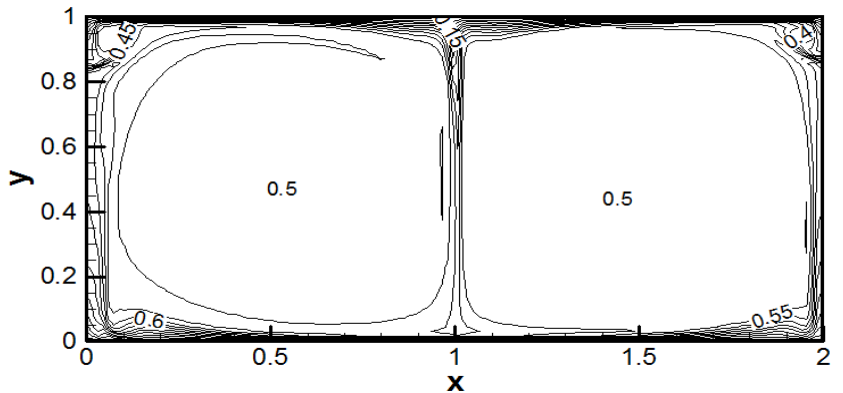

(a) $\tau=4003.594$

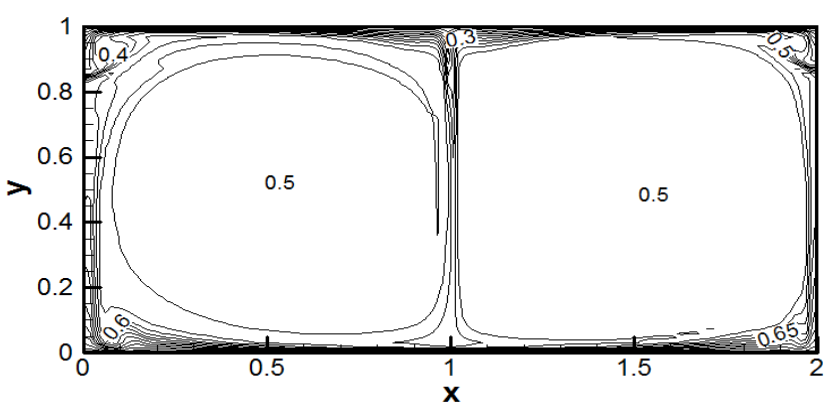

(b) $\tau=4003.994$

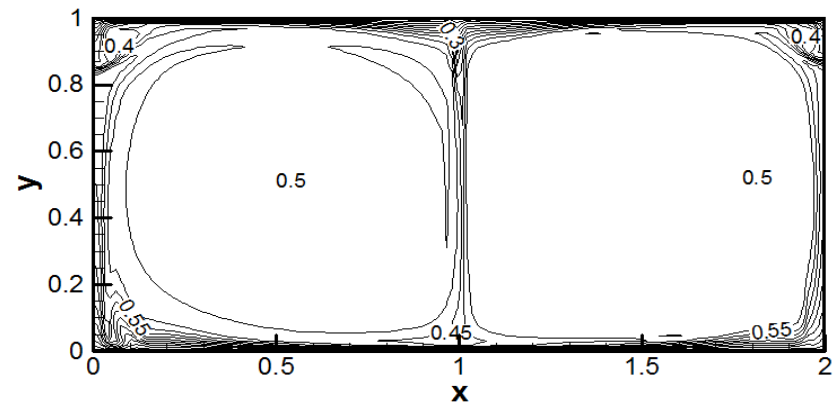

(c) $\tau=4004.394$

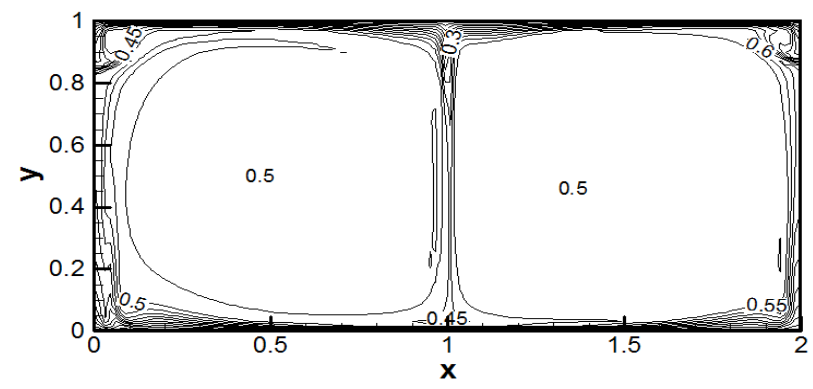

(d) $\tau=4004.794$

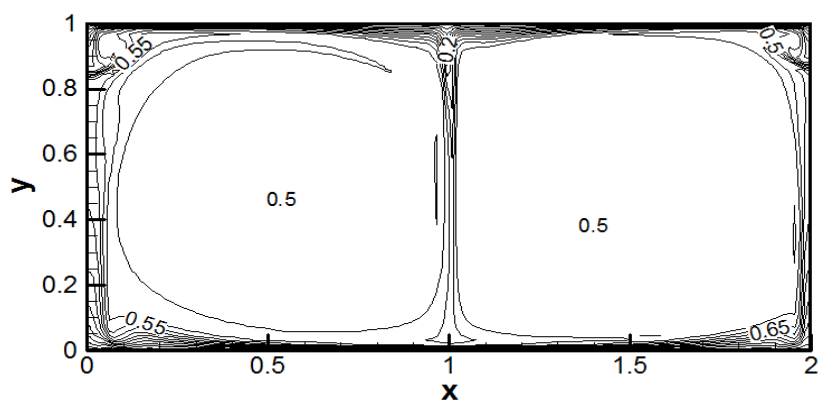

(e) $\tau=4005.594$

Fig. 6 Oscillatory solution for $\mathrm{A}=0.5, \mathrm{Nc}=1.5, \mathrm{Ra}=2000$, and $\mathrm{Sr}=\mathrm{Df}$ $=0.1, \operatorname{Pr}=0.02$ 


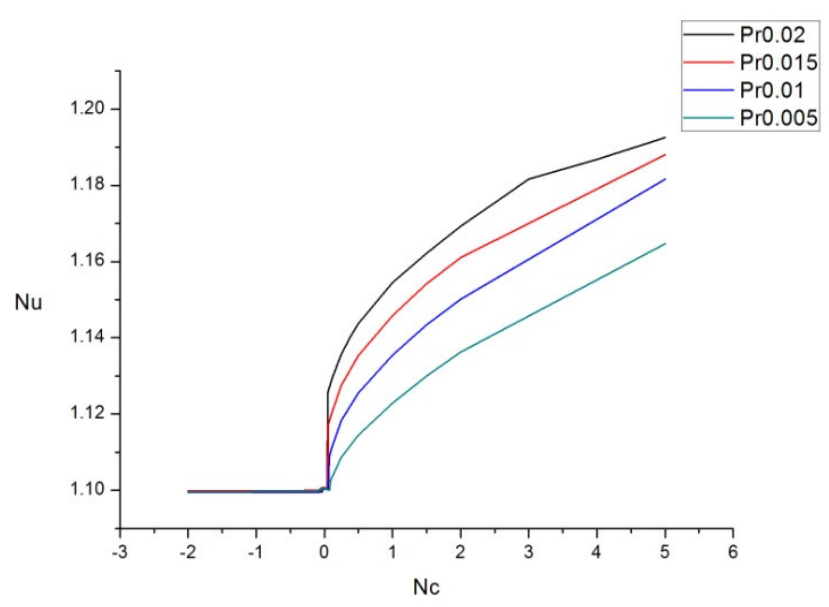

(a) average $\mathrm{Nu}$

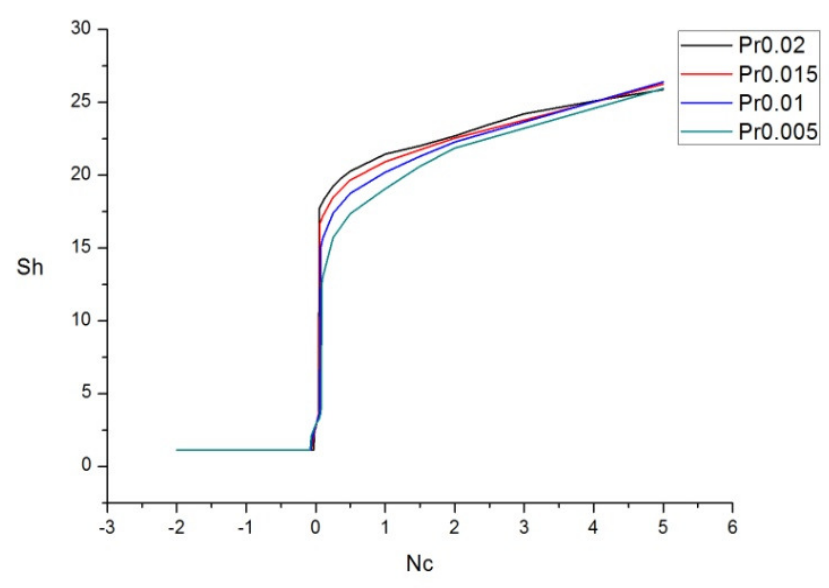

(b) average Sh

Fig. 7 Effects of buoyancy ratio on double-diffusive convection of different low Pr fluids $(\mathrm{A}=0.5, \mathrm{Ra}=2000$, and $\mathrm{Sr}=\mathrm{Df}=0.01)$

Fig. 8 presents effects of Rayleigh Number on double-diffusive natural convection in horizontal cavity under different $\mathrm{Pr}$ numbers for $\mathrm{Nc}$ $=0.5, \mathrm{~A}=0.5$ and $\mathrm{Sr}=\mathrm{Df}=0.1$. It reveals that both average Nu keeps constant firstly before first critical point, meaning the conduction plays an significant role in heat transfer. Heat transfer intensity grows stronger gradually as $\mathrm{Ra}$ increases (after that point). It exhibits a small difference of average $\mathrm{Nu}$ between the two higher Pr metal liquid fluids $(\mathrm{Pr}=0.015$ and $\mathrm{Pr}=0.02$ ). Average $\mathrm{Sh}$ grows gradually as $\mathrm{Ra}$ increases(from $\mathrm{Ra}=$ 500) before first critical point and increases faster after first critical point. Further study shows that the concentration difference between two horizontal walls intrigues the flow before first critical point. In turns, the flow enhances the mass transfer. Whereas, heat transfer remains in the conduction regime because of the weak thermal buoyancy (ie, a low Rayleigh Number ). It also can be seen that the growing trend weakens for highest $\operatorname{Pr}$ fluid $(\operatorname{Pr}=0.02)$ before the second critical point and average Sh becomes identical for different Pr fluids when Ra increases to second critical point. Further investigation shows that the flow field of fluid for $\operatorname{Pr}=0.02$ still stays in the steady convection state, while other three transform into oscillatory convection state which corresponds to high mass transfer intensity at the second critical point.

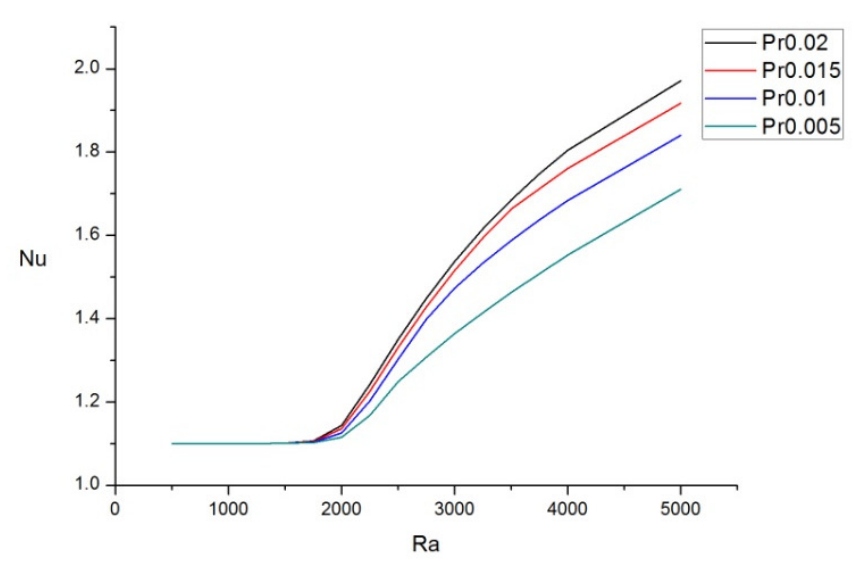

(a) average $\mathrm{Nu}$

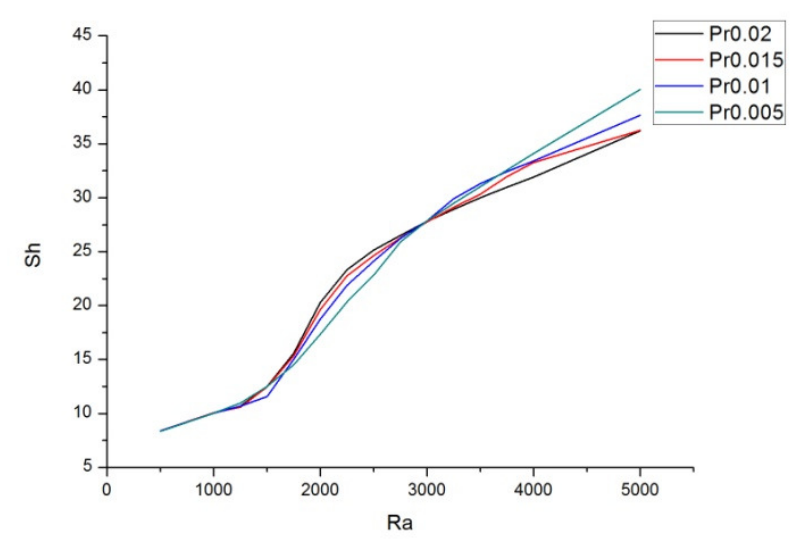

(b) average $\mathrm{Sh}$

Fig. 8 Effects of Rayleigh Number on double-diffusive convection for different low Pr fluids $(\mathrm{A}=0.5, \mathrm{Nc}=0.5$, and $\mathrm{Sr}=\mathrm{Df}=0.01)$

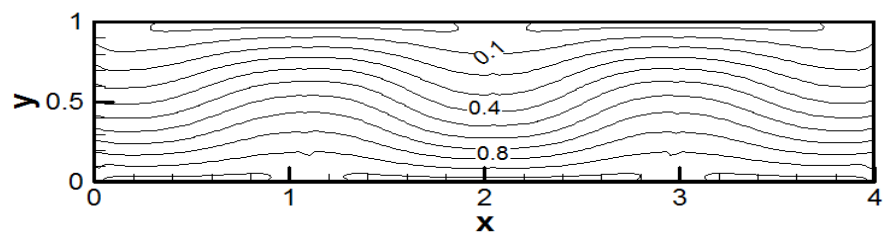

(a) contours of temperature

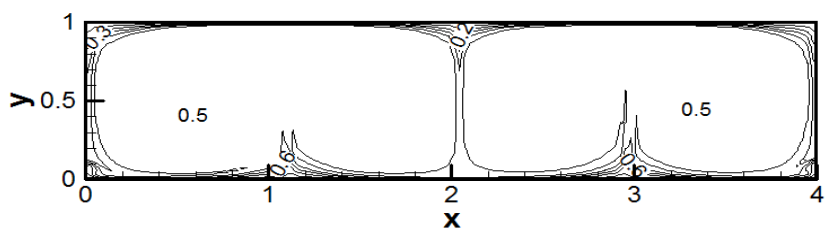

(b) contours of concentration

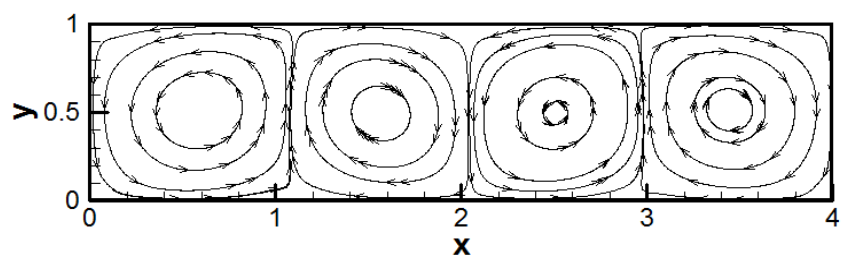

(c) flow field

Fig. 9 Convection-dominated solution for $\mathrm{A}=0.25, \mathrm{Nc}=0.5, \mathrm{Ra}=2000$, $\mathrm{Sr}=\mathrm{Df}=0.1, \mathrm{Pr}=0.02$ 
Figure 9 shows steady-state convection-dominated solutions of temperature, concentration and velocity distributions for $\mathrm{A}=0.25, \mathrm{Nc}=$ $0.5, \mathrm{Ra}=2000, \mathrm{Pr}=0.02$ and $\mathrm{Sr}=\mathrm{Df}=0.1$. Fig. 10 shows oscillatory convection-dominated solutions of temperature, concentration and velocity distributions for $\mathrm{A}=0.125, \mathrm{Nc}=0.5, \mathrm{Ra}=2000, \mathrm{Pr}=0.02$ and $\mathrm{Sr}=\mathrm{Df}=0.1(\tau=9865.225)$. For $\mathrm{A}=0.25$ and $\mathrm{Pr}=0.02$, flow exhibits steady behaviors. The vortexes develop into four vortexes compared with Fig. 5. For $\mathrm{A}=0.125$ and $\mathrm{Pr}=0.02$, flow develops into oscillatory behaviors. The vortexes change to eight vortexes compared with Fig. 5. At the same time, temperature and concentration change accordingly. These cases demonstrate that the number of vortex increases as aspect ratio A decreases. The heat and mass transfer more strongly as number of vortex increases. More cases of different $\operatorname{Pr}$ fluid $(\mathrm{Pr}=$ $0.005 / 0.01 / 0.015$ ) reveal that when aspect ratio A decreases to 0.125 , the vortexes develop into nine vortexes different from the case for $\mathrm{A}=0.125$, $\operatorname{Pr}=0.02$. The flow remains in the steady convection state for case of $\mathrm{A}$ $=0.125$, Pr $=0.005 / 0.01 / 0.015$.

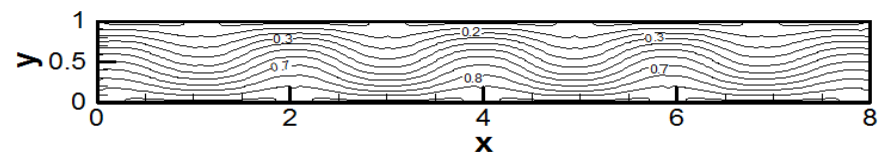

(a) contours of temperature

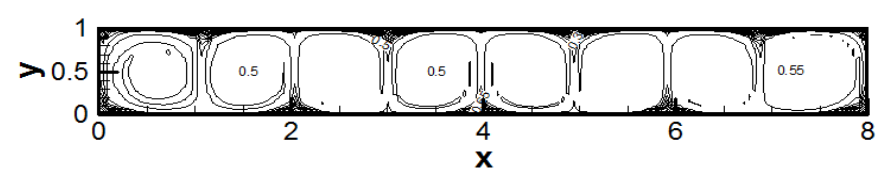

(b) contours of concentration

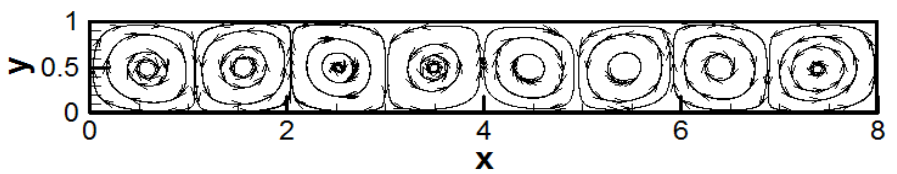

(c) flow field

Fig. 10 Oscillatory convection-dominated solution for $\mathrm{A}=0.125, \mathrm{Nc}=$ $0.5, \mathrm{Ra}=2000, \mathrm{Sr}=\mathrm{Df}=0.1, \mathrm{Pr}=0.02$

\section{CONCLUSIONS}

A unsteady numerical model for double-diffusive natural convection of $\mathrm{Pb}$-Sn alloy liquids with Soret and Dufour effects in the horizontal cavity is developed. The flow field, temperature and concentration distributions for different buoyancy ratios, Rayleigh numbers, aspect ratios under different Prandtl numbers are investigated systematically.

The results reveal that the flow structure develops from conductiondominated to convection as buoyancy ratio increases under different Prandtl numbers. It demonstrates that both heat and mass transfer intensity keep constant before the transition stage. After the transition, heat and mass transfer enhance as buoyancy ratio increases.

It can be shown from the results that heat transfer intensity keeps constant and mass transfer intensity grows slowly before first critical point as Rayleigh number increases under different Prandtl numbers. Then heat transfer intensity enhances dramatically and mass transfer intensity increases faster as Rayleigh number increases after first critical point.

The vortex number of flow structure, recirculation zones of isotherm and isoconcentration contours, heat and mass transfer intensity increase as aspect ratio decreases under different Prandtl numbers.

\section{ACKNOWLEDGEMENTS}

This work is supported by Chinese National Natural Science Foundation under Grants 51476103.
Akour, S. N., and Jarrah, M. A., 2005, "Experimental and Numerical Analysis of Natural Convection for Al-5.5\% Cu Alloy," Journal of Materials Processing Technology, s 164-165(20), 1479-1486. https://dx.doi.org/10.1016/j.jmatprotec.2005.02.142

Béghein, C., Haghighat, F., and Allard, F., 1992, "Numerical Study of Double-Diffusive Natural Convection in a Square Cavity," International Journal of Heat \& Mass Transfer, 35(4), 833-846. https://dx.doi.org/10.1016/0017-9310(92)90251-M

Bucchignani, E., and Mansutti, D., 2000, "Horizontal Thermal Convection in a Shallow Cavity: Oscillatory Regimes and Transition to Chaos," International Journal of Numerical Methods for Heat \& Fluid Flow, 10(2), 179-195. http://dx.doi.org/10.1108/09615530010312338

Campo, A., Manca, O., and Morrone, B., 2006, "Numerical Investigation of the Natural Convection Flows for Low-Prandtl Fluids in Vertical Parallel-Plates Channels," International Conference on Microelectronics, Vol.43, 572-575, IEEE Xplore. https://dx.doi.org/10.1115/1.1991867

Cao, Y., and Faghri, A., 1990, "Heat transfer in liquid metals by Natural Convection," International Journal of Heat \& Mass Transfer, 33(6), 1367-1370.

https://dx.doi.org/10.1016/0017-9310(90)90267-X

Cless, C., and Prescott, P., 1996, "Effect of Time Varying Thermal Boundary Conditions on Oscillatory Natural Convection of a LowPrandtl-Number Fluid," Numerical Heat Transfer, 29(7), 645-669. http://dx.doi.org/10.1080/10407789608913812

Efanov, A. D., Sorokin, A. P., Ivanov, E. F., Bogoslovskaya, G. P., Ivanov, V. V., Volkov, A. D., Sorokin, G.A., and Zueva, I.R., 2007, "Heat Transfer under Natural Convection of Liquid Metal during Its Boiling in a System of Channels," Thermal Engineering, 54(3), 214-222. https://dx.doi.org/10.1134/S0040601507030081

El-Genk, M. S., and Tournier, J. M. P., 2011, "Uses of Liquid-Metal and Water Heat Pipes in Space Reactor Power Systems," Frontiers in Heat \& Mass Transfer, 2(1).

https://dx.doi.org/10.5098/fhp.v2.1.3002

Eslamian, M., and Saghir, M. Z., 2011, "Thermodiffusion (Thermomigration) and Convection in Molten Semiconductor-Metal Layers," International Journal of Thermal Sciences, 50(7), 1232-1242. https://dx.doi.org/10.1016/j.ijthermalsci.2011.02.014

Hyun, M. T., Kuo, D. C., Bergman, T. L., and Ball, K. S., 1995, "Direct Simulation of Double Diffusion in Low Prandtl Number Liquids." Numerical Heat Transfer, 27(6), 639-650.

https://dx.doi.org/10.1080/10407789508913723

Jafar - Salehi, E., Eslamian, M., and Saghir, M. Z., 2014, "Estimation of molecular and thermodiffusion coefficients for Non-Ideal Molten Metal Alloys and Its Implication in Solidification Process," Canadian Journal of Chemical Engineering, 92(7), 1314-1324.

https://dx.doi.org/10.1002/cjce.21972

Kang, S., Ha, K. S., Kim, H. T., Ji, H. K., and Bang, I. C., 2013, “An Experimental Study on natural convection heat transfer of Liquid Gallium in a Rectangular Loop," International Journal of Heat \& Mass Transfer, 66(6), 192-199.

http://dx.doi.org/10.1016/j.ijheatmasstransfer.2013.07.026

Kang, S., Park, S. D., Kim, I. G., and Bang, I. C., 2016, "Numerical Study of In-Vessel Retention under the Gallium-Water External Reactor Vessel Cooling System Using MARS-LMR,"Journal of Nuclear Science \& Technology, 53(3), 345-352.

http://dx.doi.org/10.1080/00223131.2015.1045951

\section{REFERENCES}


Karcher, C., Kolesnikov, Y., Andreev, O., and Thess, A., 2002, "Natural Convection in a Liquid Metal Heated from above and Influenced by a Magnetic Field," European Journal of Mechanics, 21(1), 75-90. https://dx.doi.org/10.1016/S0997-7546(01)01164-5

Li, Y. H., Lin, K. C., and Lin, T. F., 1997, "Computation of Unstable Liquid Metal Convection in a Vertical Closed Cylinder Heated from the Side and Cooled from above," Numerical Heat Transfer, 32(3), 289-309. http://dx.doi.org/10.1080/10407789708913892

Li, Z., Yang, M., and Zhang, Y., 2016, "Double MRT Thermal Lattice Boltzmann Method for Simulating Natural Convection of Low Prandtl Number Fluids," International Journal of Numerical Methods for Heat \& Fluid Flow, 26(6), 1889-1909. https://dx.doi.org/10.1108/HFF-04-2015-0135

Ma, K., and Liu, J., 2007, "Heat-Driven Liquid Metal Cooling Device for the thermal Management of a Computer Chip," Journal of Physics D Applied Physics, 40(15), 4722-4729.

https://dx.doi.org/0.1088/0022-3727/40/15/055

Mahfooz, S. M., Hossain, M. A., and Gorla, R. S. R., 2012, "Radiation Effects on Transient Magnetohydrodynamic Natural Convection Flow with Heat Generation," International Journal of Thermal Sciences, 58(58), 79-91.

https://dx.doi.org/10.1016/j.ijthermalsci.2012.03.002

Man, Y. H., and Mi, J. J., 2000, "A Numerical Study on ThreeDimensional Conjugate Heat Transfer of Natural Convection and Conduction in a Differentially Heated Cubic Enclosure with a HeatGenerating Cubic Conducting Body," International Journal of Heat \& Mass Transfer, 43(23), 4229-4248. https://dx.doi.org/10.1016/S0017-9310(00)00063-6

Moh, J. H., Bergman, T. L., and Kuo, D. C., 1997, "Simulation of TwoDimensional, Low-Pr Natural Convection in Harmonically Oscillated, Differentially Heated Enclosures," Numerical Heat Transfer, 31(1), 1-19.

\section{http://dx.doi.org/10.1080/10407789708914022}

Prescott, P. J., and Incropera, F. P., 1994, "Convective Transport Phenomena and Macrosegregation during Solidification of a Binary Metal Alloy. I. Numerical Predictions," Journal of Heat Transfer, 116:3(3), 742-749.

https://dx.doi.org/10.1115/1.2910930

Sharma, A. K., Velusamy, K., and Balaji, C., 2009, "Turbulent Natural Convection of Sodium in a Cylindrical Enclosure with Multiple Internal Heat Sources: a Conjugate Heat Transfer Study," International Journal of Heat \& Mass Transfer, 52(11), 2858-2870.

https://dx.doi.org/10.1016/j.ijheatmasstransfer.2008.11.021

Siddiqa, S., Abrar, M. N., Hossain, M. A., and Gorla, R. S. R. 2017, "Double Diffusive Natural Convection Flow over a Wavy Surface Situated in a Non-Absorbing Medium," International Journal of Heat \& Mass Transfer, 109, 200-208.

http://dx.doi.org/10.1016/j.ijheatmasstransfer.2017.01.087

Wang, J., Yang, M., and Zhang, Y., 2015, "Coupling-Diffusive Effects on Thermosolutal Buoyancy Convection in a Horizontal Cavity," Numerical Heat Transfer Part A Applications, 68(6), 583-597. http://dx.doi.org/10.1080/10407782.2014.994412

Yin, H., and Koster, J. N., 1999, "In Situ Observation of Concentrational Stratification and Solid-Liquid Interface Morphology during Ga-5\% In Alloy Melt Solidification," Journal of Crystal Growth, 205(4), 590-606. https://doi.org/10.1016/S0022-0248(99)00262-6

Zhang, X., and Angeli, D., 2011, "Flow Transitions in a Joule-Heated Cavity of a Low-Prandtl Number Fluid," International Journal of Thermal Sciences, 50(11), 2063-2077. https://dx.doi.org/10.1016/j.ijthermalsci.2011.06.008 